\title{
TISSUE LOCALIZATION OF MULTIPLE FORMS OF ENZYMES ${ }^{\circ}$
}

\author{
George W. Nace \\ Department of Zoology, University of Michigan, \\ Ann Arbor, Mich.
}

The species specificity of enzymes and the organ specificity of isozyme combinations is no longer questioned. The attributes of tissue, cellular and sub-cellular specificity of enzymes, however, are quite uncertain. Organ specificity could, and probably does, arise as a result of any of several modes of enzyme distribution at these lower levels of organization. Thus, organ specific isozyme patterns may arise if each isozyme is characteristic of a given tissue or cell type, since organs differ from each other by the ratio and selection of their component tissue and cell types. Alternatively, these patterns may arise if more than one isozyme is found in each tissue or cell type.

These possibilities imply quite different mechanisms for the genetic control of isozymes. If one isozyme corresponds to one cell type, only a single gene-to-protein sequence need be functional in a given cell; and the different isozymes of different cell types could represent either the modulation of the action of the single locus by subsidiary genetic or cytoplasmic factors, or the action of different loci. If, on the other hand, a cell contains more than one isozyme, either a single locus must form a precursor substance whose final forms are determined by extra-chromosomal factors in the sub-cellular environment (S. Allen, 1961; Levinthal et al., 1962), a multiplicity of genetic loci must function in a given cell type as implied by the polymerization concept (Cahn et al., 1962), or a combination of these mechanisms must be operative.

At the sub-cellular level, any of several possibilities of isozyme distribution would be compatible with the several possible modes of isozyme distribution and production at the tissue and cellular levels. For example, a given isozyme might be unique to a given type of sub-cellular structure, or it might be localized on different sub-cellular structures in different cell types.

Current data do not allow selection among the various possibilities outlined above or were obtained by techniques that are not generally applicable. Mechanical separation, in quantity, of the tissues comprising complex organs lacks the necessary precision and does not seem to have been attempted. Pure line culture of various tissue or cell types is possible. Tsao

\footnotetext{
- This investigation was supported by a PHS research grant RG-5409 of the National Institutes of Health, Public Health Service, by Faculty Research Grants Nos. 34 and 441 of the Horace Rachham School of Graduate Studies, University of Michigan and by American Cancer Society Institutional Grant IN -40 .

f I wish to thank T. Tachibana, T. Suyama and T. Iwata, Marie Coon and Sue Harris for their assistance in these studies.
} 
(1960) and Caster and Prince (in press) have found several members of isozyme series in such cultures. But, it is difficult to relate these results to conditions in the intact organism because cells are notorious for their changes in culture. S. Allen (1961) has examined genetically defined lines of Tetrahymena pyriformis, and has shown that they contain multiple forms of esterases. As a first approximation, however, the protistan organism should probably be compared with the metazoan organism rather than with its constituent cells. J. Allen (1961) has taken advantage of physiological and substrate differences in the application of cytochemical procedures to show that different forms of lactate dehydrogenase (LDH) may be found at different locales within the epithelial cells of mouse epididymis. However, Allen notes that the experimental manipulations may distinguish between different, closely related isozyme sets rather than between members of an isozyme set. Nace et al., (1961) have found four electrophoretically identified $\mathrm{LDH}$ variants in a single frog oocyte. However, this observation may not have general significance in the context of the current discussion, because an egg is a unique type of cell which receives many of its macromolecular components, perhaps including some forms of $\mathrm{LDH}$, from the maternal organism.

In sum, the available evidence suggests that a given tissue or cell type does contain more than one enzyme variant, but the generality of these observations is uncertain. Moreover, the examples cited above are special cases and the procedures used do not offer general approaches to the problem of the tissue and cellular specificity of isozymes.

\section{The Echo Technique}

My coworkers and I have attempted to approach a more general solution by the combination of agar diffusion and immunohistology in a procedure shown in FIGURE 1 , and the accompanying protocol (Nace and Suyama, 1961; Nace et al., 1961). The basic requirement of this procedure, as applied to isozymes, is that antibodies directed against enzymes be available. To determine this, we have used cytochemically stained agar diffusion and immunoelectrophoretic patterns to demonstrate the existence of antibodies for acid and alkaline phosphatase and a number of dehydrogenases in complex antisera produced by rabbits, which were injected with crude homogenates (Nace and Tachibana, 1958; Nace and Alley, 1961; Nace et al., 1961; Nace and Tachibana, in press ). Figure 2 illustrates such a pattern.

Clearly, these complex antisera could not be used directly for critical immunohistological analyses. The preparation of enzymes of sufficient antigenic purity to avoid the development of any antibodies to undesired antigens seemed impractical, because the need to develop fractionation methods for each isozyme requires elaborate resources, and because any fractionation can be expected to modify the molecule in some manner. More important, as illustrated in FIGUnE 2, the stained patterns showed that non-precipitating antibodies are present in many antisera. Indeed, a 


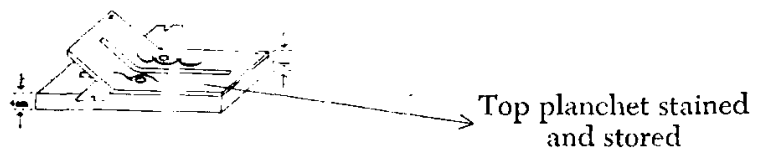

Immunoclectrophoretic or Ouchterlony plate made with fluorescent antibody at $2-3^{\circ}$ globulin concentration

Bottom sliced 3-4 times and "line" cut from agar with razor blade knife

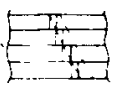

Yes

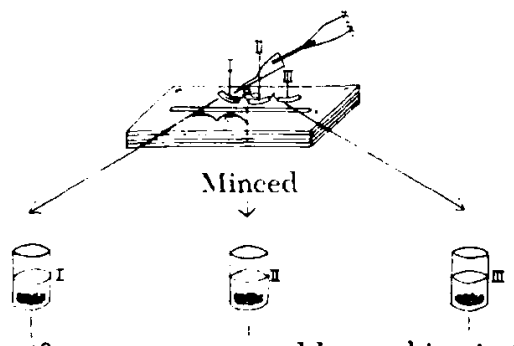

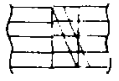

No

Uncombined and non-specific reagents removed by washing in 2-3 changes of $0.02 \mathrm{ml}$ saline for 2 hours

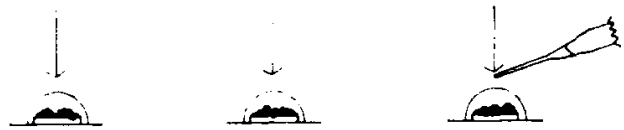

Washed, minced agar containing specific $\mathrm{AgAb} \bullet \mathrm{Fl}$ aggregate placed on fixed tissue sections

Dissociation:

a) $0.01 \mathrm{ml} ., 0.1 \mathrm{M}$ borate-carbonate $-p \mathrm{H} 10.5$

or

b) $0.01 \mathrm{ml}, 0.1 \mathrm{M}$ acetic acid-Na acetate $-p \mathrm{H} 3.5$

Diffusion:

$$
\left(\mathrm{Ag}, \mathrm{Ab} \bullet \mathrm{Fl}_{\mathbf{z}}\right)_{x} \underset{p \mathrm{H} 3.5}{\stackrel{p \mathrm{H} 10.5}{\longrightarrow} \mathrm{Ag}}+\mathrm{Ab} \bullet \mathrm{Fl}+\left(\mathrm{Ag}_{\mathrm{v}} \mathrm{Ab} \bullet \mathrm{Fl}_{\mathbf{w}}\right)_{\mathrm{u}}
$$

$22^{\circ} \mathrm{C}$., $2 \mathrm{hr}$., in humid chamber

diffusion of $\mathrm{Ab} \bullet \mathrm{Fl}$ into tissue sections

Recombination:

a) $0.01 \mathrm{ml}, 0.14 \mathrm{M} \mathrm{HCl}$

or

b) $0.01 \mathrm{ml} ., 0.1 \mathrm{M} \mathrm{NaOH}$

$22^{\circ} \mathrm{C}$., $1 \mathrm{hr}$.; Ab•Fl combines with $\mathrm{Ag}$ from agar

and with $\mathrm{Ag}$ fixed in tissue.

Washing:

30-60 min. to remove uncombined $\mathrm{Ag}$ and $\mathrm{Ab}$, unfixed $\mathrm{AgAb}$ aggregates and agar.

Note: Unfixed $\mathrm{AgAb}$ aggregates arise from the complexing of free $\mathrm{Ag}$ and $\mathrm{Ab}$ in the drop. These aggregates are trapped by the agar spread on the tissue. If they settle on the tissue they are harder to remove, but will not be in the focal plane of the tissue. Any free antigen which diffuses into the tissue and is trapped there by complexing with $\mathrm{Ab}$ can be distinguished from tissue Ag. The distribution of this free $\mathrm{Ag}$ is diffuse in character and is unrelated to the histology of the section, whereas the tissne Ag shows characteristic localization.

Mounting:

Buffered glycerin, $p \mathbf{H} 7.1$ or Elvanol (Rodriguez \& Deinhardt, '60).

Visualization:

Standard fluorescence microscopy.

Figure 1. The Echo Technique. 


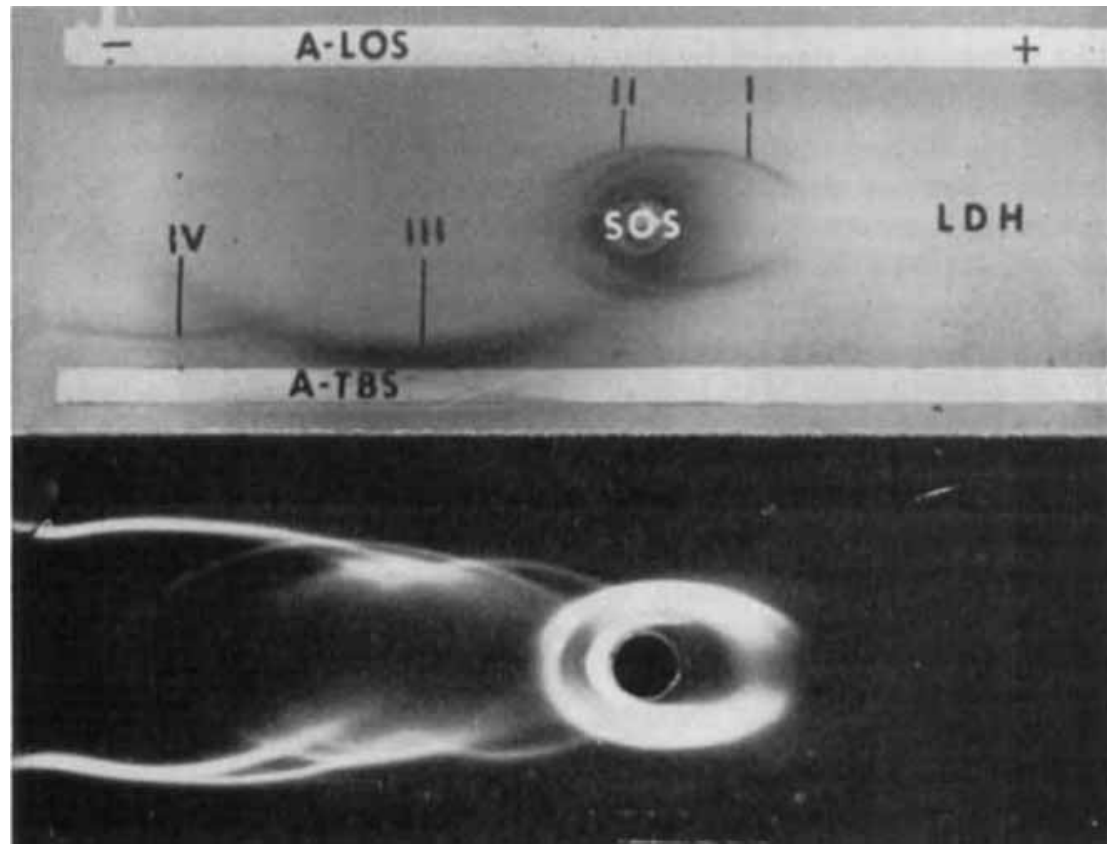

Frgure 2. An immunoelectrophoretic pattern of Rana pipiens antigens stained for lactic dehydrogenases. Center well-summer ovary supernatant (SOS). Electrophoresis: $1 \mathrm{hr} ., 17 \mathrm{~V} / \mathrm{cm}, 0.05 \mathrm{M}$ sodium veronal buffer, $p \mathrm{H} 8.6,4^{\circ} \mathrm{C}$. Upper trough-rabbit antiserum to mature ovary supernatant $(A \bullet L O S)$. Lower trough-rabbit antiserum to tailbud embryo (st. 18-19) supernatant (A•TBS). Upper figure-a planchet stained for LDH, bright field photography. Lower figure-an unstained planchet from the same plate, darkfield photography (Nace and Alley, 1961).

Lines labeled I-IV and the stained lines symmetrical to them were produced by LDH isozymes. This A-LOS did not contain antibody to LDH-III. The absence in the lower figure of a precipitation line corresponding to the LDH-III line in the upper figure reveals that this stained region represents the reaction of a non-precipitating antigen-antibody system.

reaction system showing three precipitation lines was found to contain five non-precipitating bands by treating the agar planchets with sheep antirabbit serum (Nace, unpublished). Special procedures must, therefore, be used to prove that a given antiserum contains antibodies to only one antigen, even if the antigen preparation seems to be homogeneous by all other criteria.

Since only very small quantities of fluorescent antibody are needed to demonstrate the presence of an antigen in a tissue section, we devised the procedure shown in FIGURE 1 in hope of recovering the antibody from specific lines in agar diffusion plates. This would permit separating a given antibody from coexisting antibodies, be they precipitating or non-precipitating. We call this the Echo technique because our first successful results were obtained on the day Echo I was placed in orbit, and because the use 
of the antibody is repeated. Colored illustrations of frog oocyte and oviduct preparations stained by this procedure for three isozymes of lactate dehydrogenase, are shown in Nace et al., (1961).

Our results and results obtained by others (Takeuchi, 1961) using this method demonstrate the feasibility of the procedure. However, the difficulties that are encountered suggest that the preliminary tests, controls, and precautions, be presented in greater detail.

Preliminary immunohistological tests. Prior to making an Echo analysis, each antiserum which forms good lines in agar diffusion patterns should be tested on sections of its homologous tissue. Failure to localize indicates that the histological procedures were inadequate or that, in the process of preparation, the injection antigens were modified from their native form. Positive localizations are, however, no assurance that each antibody in the complex antiserum is responsible for any localization. Some antigens will withstand paraffin embedding, others require frozen section techniques. We most frequently use acetone, acid alcohol, Carnoy's, and 10 percent formalin as fixatives.

Stability of tissues and antisera. Tissue sections should be exposed to the $\mathrm{pH}$ extremes, incubation and washes, followed by the complex antiserum to test the histological and antigenic stability of the tissues under these conditions. Modifications based on these tests minimize the loss of sections when the definitive tests are run. Failure of localization, or differences between the localization patterns seen in these sections and sections directly treated with the antiserum, indicate the lability of some or all of the antigens under the dissociation and recombination conditions selected. We have not yet found the conditions appropriate to all antigens in a given diffusion pattern, e.g., frog lysozyme (Nace et al., in manuscript).

Fluorescent antibody should also be exposed to the same sequence of changes followed by its application to fresh tissue sections. This tests the stability of the antibody.

Agar diffusion patterns. Sharp, well separated lines, and maximal antibody in the lines, are the desiderata for the agar diffusion patterns. Ouchterlony patterns are most economical of reagents. However, immunoelectrophoretic patterns may be needed to separate the lines adequately. Figure 2 illustrates an immunoelectrophoretic pattern in which the lines are not adequately separated. The use of frog serum (or some other heterologous antigenic mixture) for preliminary absorption of the antisera used for this test would have further simplified the pattern and separated the lines. Correct adjustment of initial antigen-antibody ratios is needed to assure sharp lines when the diffusion is complete. Maximal antibody in the lines is obtained by allowing diffusion to proceed to completion, thus forming the 'mature' patterns discussed by Nace and Tachibana (in press). This may require diffusion in micro-plates for 4 to 10 days at $4^{\circ} \mathrm{C}$.

Vertical lines are most readily dissected from the agar. If the lines are inclined, the dissection pattern illustrated to the left in Figure 1 should be 
followed. Such inclined lines usually indicate that the wells were allowed to dry during the diffusion period, that partially dried wells were recharged with reagents, or that the agar was allowed to dry from its upper surface prior to introducing the reagents. All such drying must be avoided, because it leads to absorption rather than diffusion of the reagents into the agar.

Washing the dissected agar. After agar containing the desired line is dissected from the plate, it is minced or disrupted by freezing and thawing, and is then washed free of contaminants. This washing is conveniently done in a drop inside a small siliconized Petri dish. The mincing and washing is controlled by similarly treating fragments of agar from diffusion plates which contained fluorescent antiserum but no antigen, and from diffusion plates which contained fluorescent pre-injected serum. Though not a requirement, the washing is simplified if the antiserum has been fractionated for globulin and is also absorbed.

Dissociation and recombination of antibody. Tissue sections are mounted on coverslips and are protected from desiccation in small Petri dishes lined with damp filter paper. The area around the tissue is wiped with siliconized paper to limit the fluid drop to the area of the tissue; and the minced agar containing the specific precipitate is spread evenly over the surface of the tissue to protect it from antigen-antibody aggregates which will settle out of the drop during the recombination phase of the staining.

The $p \mathrm{H}$ of the tissue-agar combination is adjusted with buffer chosen on the basis of preliminary tests on intact diffusion patterns. The correct $p \mathrm{H}$ and buffer will cause the appropriate line in the agar to disappear. The molarity of this buffer should be adjusted to avoid excessive dilution of the drop covering the tissue section and care must be taken to minimize the loss of material when determining the $p \mathrm{H}$ with indicator paper. Similar precautions are taken during the recombination phase. Excessive dilution may be corrected by allowing controlled evaporation from the drop.

Washing the tissue section. On completion of the dissociation, diffusion, and recombination phases, the agar and antigen-antibody aggregates which form in the drop must be removed. This is done by placing the coverslip in racks hung on a rocking arm that gently carries the sections through the distilled water, saline, or buffer washes appropriate to each tissue. This is a critical phase of the procedure, since incomplete washing will leave the tissue covered with debris that hinders detailed observation and photography, while excessive washing may loosen the tissue from the coverslip. Diffusion and recombination phases of the procedure are conducted at room temperature to avoid the fusion of agar and tissue which occurs at higher temperatures.

Additional controls and procedures. Plates which contain antiserum but no antigen, which contain fluorescent pre-injection serum, and which contain no components of the immune system provide pieces of agar which are carried through all steps in the procedure to control washing, auto- 
fluorescence and the possibility that some of the inorganic reagents contain fluorescing contaminants.

Tests with the agar which contained free antiserum are also prepared without washing the agar. These tests confirm the stability of the system to the various treatments, demonstrate whether or not the dissociation step was effective, and indicate whether or not sufficient time was allowed for the antibody to diffuse from the agar into the tissue.

Tests with unwashed agar, containing the fluorescent pre-injection serum, reveal the extent of possible nonserological binding of the conjugate; a source of fluorescence which plagues the usual immunohistological work. The Echo technique, however, is essentially free of this difficulty because non-specifically binding substances are removed when the agar containing the specific precipitate is washed.

We have had some success using plates prepared with non-fluorescent antiserum which is then made visible with fluorescent sheep antirabbit serum. This procedure simplifies the preparation of the original agar plates, but the additional treatment and washing of the sections increases the danger of losing the tissue.

Observational problems. If an antigen or other cytochemically detected molecule is found in high concentration at a few sites in a tissue, it may be readily identified. However, if it is present in low concentration at dispersed sites, as would be expected for many enzymes, it may be extremely difficult to distinguish from artefacts. This problem is accentuated by the great specificity of the Echo technique and is only mitigated by careful washing and critical controls. For this reason, many of our results which secm interesting must be further confirmed before they can be reported.

\section{LDH Isozymes and the Echo Technique}

One of our objectives with the Echo technique is to examine the tissue and cellular localization of isozymes. This requires that antigenic differences exist between the enzyme variants. Cahn et al., (1962) report that the most cathodally migrating (CM) and the most anodally migrating (CH) forms of chicken LDH are antigenically distinct, but that the intermediate forms show varying degrees of cross reactions with antisera to each of the extreme types. On this and other evidence Cahn et al. concur with Markert and his coworkers $(1961 ; 1962)$ who hypothesized that the several forms of LDH are polymers of two distinct monomers. Cahn et al., (1962) did not report the number of antisera capable of distinguishing between $\mathrm{CM}$ and $\mathrm{CH}$ while reacting with the intermediate forms, the number of antisera which should have but did not show this spectrum of specificity, or any results with antisera produced by injecting the intermediate variants.

Our antisera to frog embryo and organ supernatants usually contain antibodies directed against one or two forms of LDH. However, antisera have been obtained which produced as many as four lines which stained as LDH. FIGURE 2 illustrates a pattern produced by such antisera. It will be noted that the several LDH arcs in these patterns cross each other. 
This is indicative of antigenic differences. If the only distinct antigens were those at the cathodal and anodal extremes, the intermediate, cross-reacting forms of Cahn el al., (1962) would cause these arcs to fuse and forms a continuous, wavy line. Our patterns indicate, therefore, that these enzymes do possess distinctive antigenic properties and we conclude that either the isozymes are distinct molecules which share a common enzymatically active moiety or that, as suggested by Cahn et al. (1962), the arrangement of the monomers does determine the antigenic specificity of the LDH molecules.

The results of Echo analysis of these lines on frog oviduct (Nace and Suyama, 1961; Nace et al., 1961) were that; our LDH I stained the connective tissue on the peritoneal side of the oviduct; that LDH II stained only some cells in the ciliated epithelial lining of the oviduct; and that LDH III stained some cells in this epithelium, but also the glandular portion of the oviduct. Note that LDH II and III may or may not stain the same or adjacent cells in the epithelium. We have not yet resolved the LDH IV line from an adjacent nonenzyme line, therefore, it has not bcen tested. This pattern of localization confirms the immunoelectrophoretic evidence that these forms of LDH are antigenically distinct, or we would have obtained identical localizations for each of them. This follows, since LDH I is probably the $\mathbf{H}$ form of Cahn et al. (1962), and LDH II and III are among the intermediate forms.

Why Cahn et al., (1962) and Markert (1962) find cross-reactions among the LDH isozymes, whereas I do not, cannot be readily explained. It must be noted, however, that they used birds and mammals, respectively, while I used an amphibian. It is also possible that their fractionation procedures stripped labile groups responsible for specificity from the molecules, at the same time exposing common reactive sites.

These results suggest: that within an organ some isozymes may be found in only a single tissue type, (e.g., LDH I in connective tissue, and LDH II in epithelium); that some may be found in several tissue types, (e.g., III in epithelium and jelly gland); and that a given tissue may contain more than one isozyme (e.g., the oviduct epithelium contains LDH II and III).

\section{Summary}

The several possible modes of tissue, cellular, and subcellular distribution of multiple forms of enzymes suggest that several different mechanisms controlling isozyme formation must be envisioned. The choice among these mechanisms requires a knowledge of isozyme distribution that is not readily acquired. A technique for this purpose which combines agar diffusion and immunohistological procedures is presented in detail.

With this technique it was found that frog lactic dehydrogenase isozymes show several of the possible modes of distribution. The agar diffusion and immunohistological data also indicate that the several forms of lactic dehydrogenase can be distinguished antigenically. 


\section{References}

Altes, J. M. 1961. Multiple forms of lactic dehydrogenase in tissues of the mouse: Their specificity, cellular localization, and response to altered physiological conditions. Ann. N. Y. Acad. Sci. 94 (3): 937-951.

Ar.LEN, S. L. 1961. Genetic control of the esterases in the protozoan Tetrahymena pyriformis. Ann. N. Y. Acad. Sci. 94(3): 753-773.

Appella, E. \& C. L. Markert. 1961. Dissociation of lactate dehydrogenase into subunits with guanidine hydrochloride. Biochem. Biophys. Res. Com. 6: 171-176.

Cain, R. D., N. O. Kaplan, L. Levine \& E. Zwili.ing. 1962. Nature and devclopment of lactic dehydrogenases. Science. 136: 962-969.

Caster, C. W. \& R. K. Phince. Starch gel "zymograms" of cultured connective tissue cells. Lab. Invest. In press.

Levinthal, C., E. R. Signer \& K. Fetheroff. 1962. Rectivation and hybridization of reduced alkaline phosphatase. Proc. Nat. Acad. Sci. 48: 1230-1237.

Mankert, C. L. 1962. Isozymes in kidney development. In "Proccedings of the 13th Annual Conference on the Kidney-Genctic, Developmental and Immunological Aspects of Renal Diseases." J. Metcoff, Ed. Northwestern University Press, Evanston, Ill.

Markert, C. L. \& H. Ursprung. 1962. The ontogeny of isozyme patterns of lactate dehydrogenase in the mouse. Develop. Biol. In press.

NaCe, G. W. \& T. Tachibana, 1958. The characterization of antigenic systems of the embryo by the application of cytochemical techniques to agar diffusion patterns. Anat. Record. 131: 584.

Nace, G. W., T. Suyama \& N. Smith. 1961. Early development of special proteins. In "Germ cells and Earliest Stages of Development. Symposium of I.I.E.Pallanza, 1960.” S. Ranzi, Ed.: 564-603.

Nace, G. W. \& T. Suyama. 1961. The Echo technique: A procedure for the cellular localization of specific enzymes and other antigens utilizing preliminary agar diffusion with fluorescent antibody. J. Histochem. \& Cytochem. 9: 596.

NACE, G. W. \& J. W. Alley. 1961. On the photography of unstained, differentially stained and fully stained precipitation lines in agar. J. Biol. Photo. Assoc. 29: 125133.

Nace, G. W. \& T. Tachibana. Micro-immunozymograms and the visualization of a non-precipitating antibody. Immunol. In press.

Nace, G. W., T. Suyama \& M. Coon. Lysozyme: A "deletion" encountered in studies of frog embryo, kidney and kidney adenocarcinoma antigens. In manuscript.

Ronfiquez, J. \& F. Deinhardt, 1960. Preparation of a semipermanent mounting medium for fluorescent antibody studies. Virology. 12: 317.

Takeuchi, I. 1961. See Carnegie Institution of Washington Yearbook. 60: 417. Also Personal Communication.

Tsao, M. U. 1960. Heterogeniety of tissue dehydrogenases. Arch. Biochem. Biophys. 90: $234-238$. 